\title{
Initial localization and kinematic characteristics of structure components of a CME
}

\author{
A.M. Uralov and V.V. Grechnev \\ Institute of Solar-Terrestrial Physics, Irkutsk, Russia, email: uralov,grechnev@iszf.irk.ru
}

The main observable components of coronal mass ejections (CMEs) are the core, cavity and the frontal structure (FS), which is the leading edge of the visible CME. The core of a CME is associated with an eruptive filament whose motion can be followed just from the solar surface. As a rule, the FS is visible in scattered white light (SOHO/LASCO) far from the CME origination site. In few cases in near-the-limb events, the FS was detected closer to the solar surface. However, it appears not possible to detect any manifestations of the FS in a reasonable proximity of the pre-eruptive filament localized on the sun far from the limb. The identification of the FS remains unclear. We propose a method to estimate parameters of the initial volume of a CME based on the comparison of measured height-time plots of its structural components with a self-similar solution of MHD equations describing the expansion of a CME.

We examine such a method by analyzing observations of an eruptive filament on the solar disk on September 4, 2000 using data acquired with SOHO/LASCO \& EIT, Yohkoh/SXT, and the Siberian Solar Radio Telescope. That was a large quiescent filament located beyond active regions and surrounded by weak, large-scale magnetic fields. We estimate both surface and height extent of the initial CME volume. The surface scale characterizes the size of the solar surface that determines the generation and subsequent evolution of the CME. The surface size of the CME appears to be comparable with the maximum extent of the flare ribbons. The initial acceleration of the CME is of order of some $\mathrm{km} / \mathrm{s}^{-2}$, which looks plausible. The initial location of the CME frontal structure is about $125 \mathrm{Mm}$ above the pre-eruptive filament. Such scales are comparable with coronal cavities surrounding quiescent filaments. One may therefore assume that the frontal structure in the event considered is localized above the filament, near the separatrix surface confining the coronal cavity. We also discuss a possible observable manifestation of the separatrix surface in soft X-rays.

Kinematic characteristics of the eruptive filament and CME's frontal structure are shown in figure 1. a) Experimental data. The positions of the filament are marked with small circles (SSRT) and triangles (LASCO), and those of the frontal structure are marked with squares (EIT) and crosses (LASCO). The horizontal axis shows the universal time, and the vertical axis shows the distance from the solar disk center. b) The comparison of the experimental height-time plots with the self-similar invariant kinematic plots. The vertical axis shows the dimensionless coordinate $x=R_{s} / R_{0, s}$, where $R_{s}$ is the distance measured from the virtual expansion center and $R_{0, s}$ is the initial radius of the CME. The horizontal axis shows the dimensionless time $t-t_{0} / \tau$, where $\tau=R_{0, s} / U_{\infty, s}$ is the initial CME's time scale, $U_{\infty, s}$ is the asymptotic velocity of the frontal structure, and $t_{0}$ is the onset of the self-similar expansion. c) Same as (b), initial part.

Despite the closeness of the heights of the frontal structure and the coronal cavity, the former hardly is a simple copy of the upper part of the moving separatrix surface 

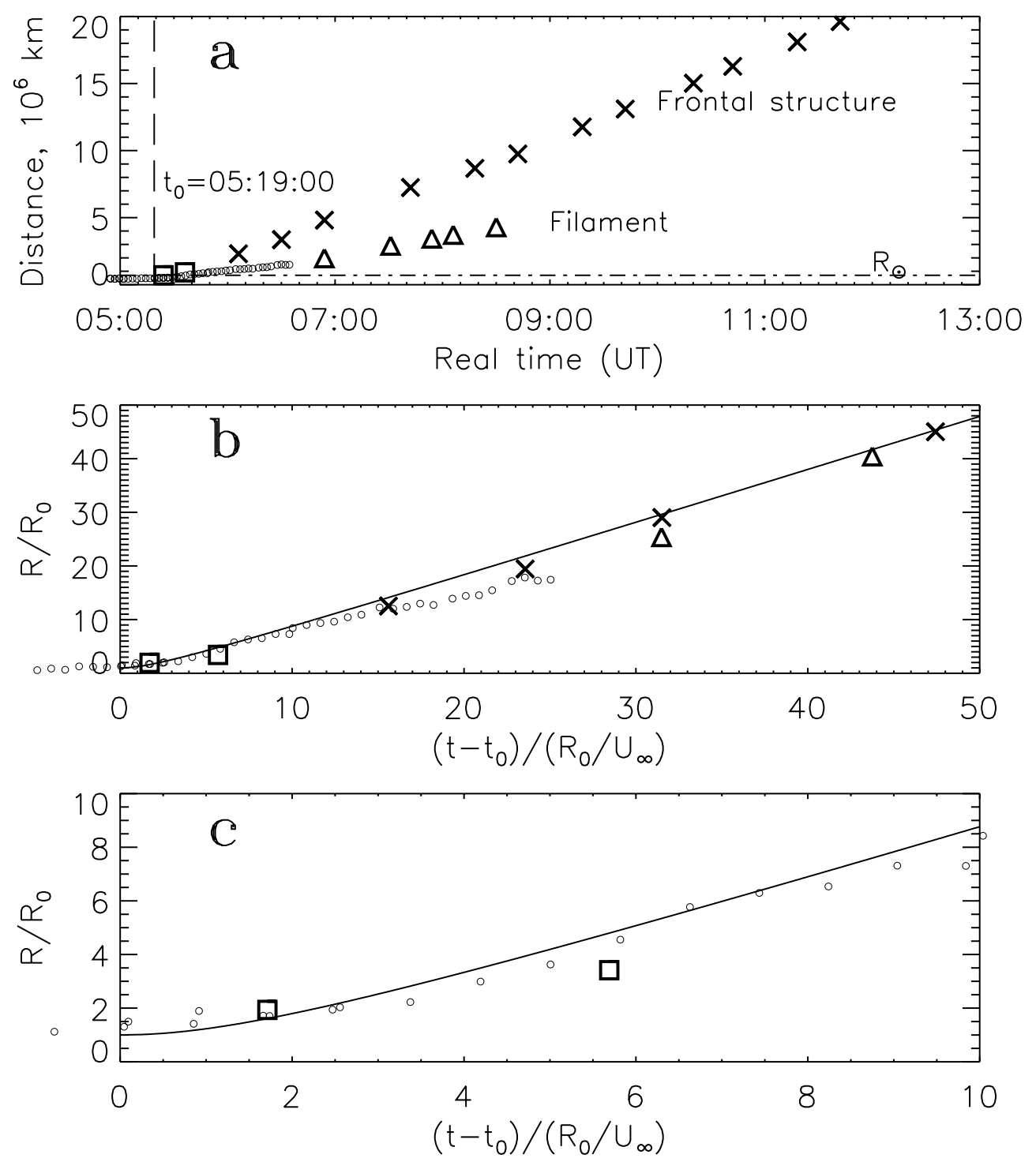

Figure 1. Kinematic characteristics of the eruptive filament and CME's frontal structure

that confines the cavity. At the initial stage of the CME expansion, the main part of the frontal structure is possibly a large-scale loop structure extended along the eruptive filament and resembling its leading edge. At least, some part of the frontal structure stuff has a temperature of order 1.5 MK at a distance of order of the solar radius from the eruption site. Its initial temperature can significantly exceed this value.

\section{Acknowledgements}

We thank the instrumental teams of the Yohkoh and SOHO for the open-data policies which made available for us data used in this study. SOHO is a project of international cooperation between ESA and NASA. Yohkoh is a project of international cooperation between ISAS and NASA. This research is supported by the Russian RFBR grant 0302-16591 and the Russian Ministry of Science grant 477.2003.2. 